\title{
CP Violation in nonleptonic two-body decays of D
} Mesons

\author{
Pietro Santorelli* \\ Università di Napoli Federico II - Dipartimento di Fisica "E. Pancini" \\ Complesso Universitario di Monte Sant'Angelo \\ I-80126 Napoli Italy \\ and \\ INFN-Sezione di Napoli, Napoli Italy \\ E-mail: pietro.santorelli@na.infn.it
}

\begin{abstract}
The nonleptonic two body decays of the $D$ and $D_{s}$ mesons represent a key subject to study possible effects of CP violation. The attention for these decay modes was triggered four years ago by the results of measurements of the difference between $\mathrm{CP}$ asymmetries in charged $K K$ and $\pi \pi$ final state, $\Delta a_{\mathrm{CP}}$. Although the measured $\Delta a_{\mathrm{CP}}$ is now compatible with zero the nonleptonic decays of charmed mesons into a pair of light mesons is an interesting subject for many reasons. Here we discuss phenomenological models to study the decays into two pseudoscalar mesons.
\end{abstract}

VIII International Workshop On Charm Physics

5-9 September, 2016

Bologna, Italy

${ }^{*}$ Speaker. 


\section{Introduction}

The study of nonleptonic decays of charmed mesons is particularly interesting for many reasons ${ }^{1}$.

- These decays give us the possibility to study the $\mathrm{CP}$ violation effects in the case of a quark of up-type. In fact the Singly Cabibbo Suppressed (SCS) decay amplitudes receive contribution from current-current operators and from penguin operators with different weak phases. This is the necessary condition to reveal CP violating effects. In fact it is possible to show that the amplitudes for SCS decay modes can be written as

$$
\mathscr{A}^{S C S}=\frac{1}{2}\left(V_{c s}^{*} V_{u s}-V_{c d}^{*} V_{u d}\right) A^{(1,2)} e^{\iota \delta}-\frac{1}{2} V_{c b}^{*} V_{u b} A^{(P)} e^{l \delta^{\prime}},
$$

where the $A^{(1,2)}$ and $A^{(P)}$ are the tree and the penguin amplitudes, respectively. While $\delta$ and $\delta^{\prime}$ are strong phases. The related CP direct asymmetry is

$$
a_{\mathrm{CP}}=\frac{|\mathscr{A}|^{2}-|\overline{\mathscr{A}}|^{2}}{|\mathscr{A}|^{2}+|\bar{A}|^{2}}=\eta A^{2} \lambda^{4} \sin \left(\delta-\delta^{\prime}\right)\left[\frac{A^{(P)}}{A^{(1,2)}}\right] \approx\left(6 \times 10^{-4}\right) \sin \left(\delta-\delta^{\prime}\right)\left[\frac{A^{(P)}}{A^{(1,2)}}\right] .
$$

The estimation of $a_{\mathrm{CP}}$ requires the calculation of the strong phases, which are expected to be large due to the presence of resonances with masses near to the mass of $D$ mesons, and hadronic amplitudes, $A^{(P)}$ and $A^{(1,2)}$.

- The calculation of the hadronic amplitudes represents a very challenging goal. The mass of the charm quark is not enough large to benefit from the powerful approach of Heavy Quark Effective Theory [4]. Neither it is possible to use the Chiral Perturbation Theory because the charm mass it too large respect the scale of the strong interactions. The only available first principle calculational method remains the lattice [5, 6], but for now this approach is not reliable for the charmed mesons. Meanwhile, these processes are studied by phenomenological models.

In the following we will consider the decays of $D$ and $D_{s}$ mesons in two pseudoscalar mesons in the framework of the Standard Model and in particular we will focus our attention on the use of $S U(3)_{F}$ symmetry. This approach dates back to the discovery of the $J / \psi$ meson $[7,8,9,10]$. However, we limit ourself to review literature on the subject after 2012, the year of the first experimental measurements of $\Delta a_{\mathrm{CP}}=a_{\mathrm{CP}}\left(D^{0} \rightarrow K^{+} K^{-}\right)-a_{\mathrm{CP}}\left(D^{0} \rightarrow \pi^{+} \pi^{-}\right)[11,12]^{2}$. The first large measured value of $\Delta a_{\mathrm{CP}}$ triggered a lot of research devoted to understand this result. There was two different and well motivated positions respect to the compatibility of the data with the Standard Model. Many authors supported the idea that direct CP violation of the order of $1 \%$ in the charm sector is a clear sign of New Physics effects [18]. Many others [19] have arguments in favor of the possibility to account for large CP violation effects in the Standard Model as a consequence of large phases coming from Final State interaction (FSI).

\footnotetext{
${ }^{1}$ See also $[1,2,3]$.

${ }^{2}$ See also $[13,14]$ and the more recent LHCb measurements $[15,16,17]$
} 


\section{The $D \rightarrow P P$ decay modes and $S U(3)_{F}$ symmetry}

The decays of $D$ and $D_{s}$ mesons into two light ones can, in principle, be studied by assuming $S U(3)_{F}$ flavour symmetry. In the case of two pseudoscalar mesons the idea is to classify the final states as the symmetric part of product of the two octets ${ }^{3}$, which classifies the pions, kaons and the $\eta_{8}$

$$
P P \sim(\mathbf{8} \otimes \mathbf{8})_{S}=\mathbf{1} \oplus \mathbf{8} \oplus \mathbf{2 7} .
$$

The $D$ mesons can be regarded as members of a anti-triplet of $S U(3)_{F},\left(D^{0}, D^{+}, D_{S}\right) \sim \overline{\mathbf{3}}$; while, being the effective hamiltonian responsible of charm decays made of three light quark fields and one charm field, it is given by the tensor product

$$
\mathscr{H} \sim \mathbf{3} \otimes \overline{\mathbf{3}} \otimes \mathbf{3} \sim \mathbf{3} \oplus \mathbf{3}^{\prime} \oplus \overline{\mathbf{6}} \oplus \mathbf{1 5}
$$

The physical amplitudes in this approach are given by

$$
\langle P P|\mathscr{H}| D\rangle \sim\langle\mathbf{1} \oplus \mathbf{8} \oplus \mathbf{2 7}|(\mathbf{3} \oplus \overline{\mathbf{6}} \oplus \mathbf{1 5})| \overline{\mathbf{3}}\rangle .
$$

The Wigner-Eckart theorem allow us to write all the amplitudes (corresponding to well defined initial and final state, they are fixed by their values of the isospin, and hypercharge operators) in terms of three unknown Reduced Matrix Elements (RME) ${ }^{4}$

$$
\langle\mathbf{8}|| \mathbf{1 5}|| \overline{\mathbf{3}}\rangle, \quad\langle\mathbf{2 7}|| \mathbf{1 5}|| \overline{\mathbf{3}}\rangle, \quad\langle\mathbf{8}|| \overline{\mathbf{6}}|| \overline{\mathbf{3}}\rangle .
$$

Therefore, in the limit of symmetry, the theoretical description of all these decay modes requires to fix 5 unknown parameters (they became 9 if we include the contribution of the 3 representation). Anyway, by looking at the experimental data it is easy to understand that the $S U(3)_{F}$ flavour symmety is strongly violated. In fact, for example, the relations [20, 21]

$$
\begin{aligned}
A\left(D^{0} \rightarrow K^{+} K^{-}\right) & =-A\left(D^{0} \rightarrow \pi^{+} \pi^{-}\right), \\
a_{C P}^{d i r}\left(K^{+} K^{-}\right) & =-a_{C P}^{d i r}\left(\pi^{+} \pi^{-}\right), \\
\tan \theta_{C} A\left(D^{+} \rightarrow \bar{K}^{0} \pi^{+}\right) & =\sqrt{2} A\left(D^{+} \rightarrow \pi^{0} \pi^{+}\right), \\
B r\left(D^{0} \rightarrow K^{+} \pi^{-}\right) & =\tan ^{4}\left(\theta_{C}\right) B r\left(D^{0} \rightarrow K^{-} \pi^{+}\right), \\
A\left(D^{0} \rightarrow K^{0} \bar{K}^{0}\right) & =0,
\end{aligned}
$$

dictated by the $S U(3)_{F}$ symmetry are at odds with data [22]. The linear breaking of the symmetry has been discussed in $[23,24]$, where the authors wrote the new operators appearing in the analysis for $m_{s} \neq m_{u, d}$ and identify 15 new RMEs. At the end if we take into account also the linear breaking of $S U(3)_{F}$, the description of the $D \rightarrow P P$ modes needs 18 RME (neglecting the contribution of the penguin operators, 3 ), while the decay channels are 17. In [23] the authors show that only 13 RMEs are independent and so the (real) free parameters in this approach (they are 25) can be fixed

\footnotetext{
${ }^{3}$ We are considering just the octect, if we want to consider also the $\eta$ and $\eta^{\prime}$ we should take into account another singlet, in our approximation we should assume $\eta=\eta_{8}$.

${ }^{4} \mathrm{We}$ are neglecting the part of the Hamiltonian transforming as $\mathbf{3}$ because it is proportional to the CabibboKobayashy-Maskawa terms $V_{c b}^{*} V_{u b}$. The inclusion of this term add two new RME.
} 
by a fit to the 17 experimental branching ratios, $8 \mathrm{CP}$ direct asymmetries and the data on the strong phase, $\delta_{K \pi}$. In this framework, all the available experimental data have been used to fix the free parameters and one cannot give any prediction. However, the compatibility with the experimental data is useful to assess the validity of the theoretical approach.

\section{The topological amplitudes approach and $S U(3)_{F}$}

As we have seen in the previous section, the linear breaking of $S U(3)_{F}$ can be consistently included into the analysis of the amplitudes at the cost of a number of real parameters larger than the number of the branching ratios. An alternative way to study these decay modes involves topological amplitudes which are characterized by the flavour flow in the decays [25, 26, 27, 28]. In [29] the $D \rightarrow P P$ modes are studied by combining topological amplitude approach and $S U(3)_{F}$ assumptions. It is shown that a map can be constructed between the topological amplitudes and the reduced amplitudes characterized by $S U(3)_{F}$ representation. This map can accommodate also the linear corrections to $S U(3)_{F}$ symmetry due to $m_{s} \neq m_{u, d}$. Moreover, to reduce the number of free parameters, the Tree $(T)$ and Annihilation $(A)$ amplitudes are evaluated in the factorization assumption by considering and fitting $1 / N_{c}^{2}$ corrections to them. Here the constraints on the strength of $1 / N_{c}$ corrections and on the maximal size of linear $S U(3)_{F}$ breaking allow to fix the 27 free parameters by fitting 16 measured branching ratios and one strong phase.

Concerning the calculation and the capability to predict CP asymmetries in this approach, we should remember that the $\mathrm{CP}$ violating part of the decay amplitudes are suppressed and so any procedure which consists in fitting branching ratios cannot determine them. Furthermore, once identified the topological amplitudes contributing to the decay amplitudes, one can construct sum rules of direct $\mathrm{CP}$ asymmetries in such a way a cancellation between the unknown amplitudes occurs. In [30] the unknown penguin $(P)$ and penguin annihilation $(P A)$ topological amplitudes disappear by conveniently combine the following $\mathrm{CP}$ asymmetries

sum rule 1: $a_{\mathrm{CP}}\left(D^{0} \rightarrow \pi^{+} \pi^{-}\right), a_{\mathrm{CP}}\left(D^{0} \rightarrow \pi^{0} \pi^{0}\right)$ and $a_{\mathrm{CP}}\left(D^{0} \rightarrow K^{+} K^{-}\right)$,

sum rule 2: $a_{\mathrm{CP}}\left(D^{+} \rightarrow K^{+} \bar{K}^{0}\right), a_{\mathrm{CP}}\left(D_{s} \rightarrow K^{0} \pi^{+}\right)$and $a_{\mathrm{CP}}\left(D_{s} \rightarrow K^{+} \pi^{0}\right)$,

a numerical analysis of the correlation between two asymmetries starting from the experimental data on the last one is done in [30].

\section{A pure topological approach to the $D \rightarrow P P$ modes}

In ref. [31] the analisys of the SCS decay modes and the corresponding CP direct asymmetries are studied by the use of the topological amplitudes approach [25, 26, 27, 28]. Possible contributing diagrams can be found in Figure 1.

The $S U(3)_{F}$ symmetry breaking effects has been considered by introducing five parameters depending on the final state. Amplitudes and SU(3) breaking parameters are fixed by fitting SCS decay modes of $D$ and $D_{s}$ mesons and the related CP asymmetries. The final states with $\eta$ and $\eta^{\prime}$ has been taken into account. We refer to the article for a detailed discussion on the fit procedure. However, by putting some constraints on the parameters as suggested by the result of the analysis on 


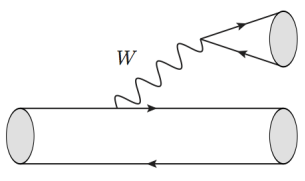

(a) $T$

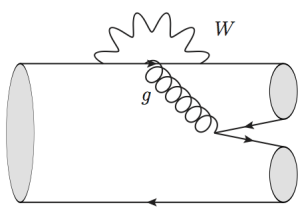

(c) $P, P_{\mathrm{EW}}^{C}$

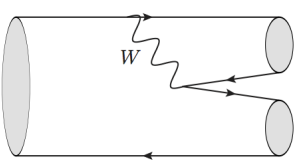

(b) $C$

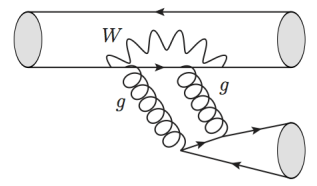

(d) $S, P_{\mathrm{EW}}$

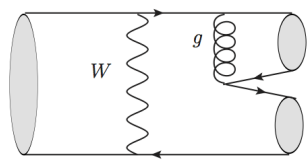

(e) $E$

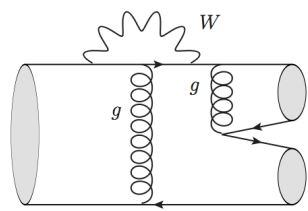

(g) $P E, P E_{\mathrm{EW}}$

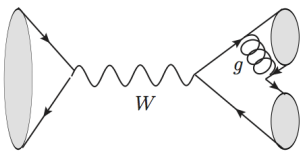

(f) $A$

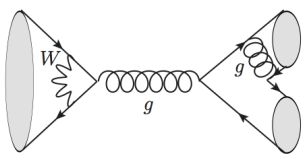

(h) $P A, P A_{\mathrm{EW}}$

Figure 1: All the possible, non negligible, flavor diagrams: (a) color allowed tree, $T$; (b) color-suppressed tree $C$; (c) QCD-penguin $P$; (d) QCD-penguin singlet $S$; (e) Exchange $E$; (f) Annihilation $A$; (g) QCDpenguin exchange $P E$; (h) QCD-penguin annihilation $P A$.

the CF in Ref. [32], in their fit case II [31] they have a smaller number of free parameters (respect to the experimental data) and so they are able to give predictions for direct $\mathrm{CP}$ asymmetries

$$
\begin{aligned}
a_{C P}\left(D^{0} \rightarrow \pi^{+} \pi^{-}\right) & =+3.3 \times 10^{-4}, \\
a_{C P}\left(D^{0} \rightarrow K^{+} K^{-}\right) & =-7.3 \times 10^{-4}, \\
a_{C P}\left(D^{0} \rightarrow \bar{K}^{0} K^{0}\right) & =-5.8 \times 10^{-4}, \\
a_{C P}\left(D^{0} \rightarrow \pi^{0} \eta\right) & =-4.7 \times 10^{-4}, \\
a_{C P}\left(D^{0} \rightarrow \pi^{0} \eta^{\prime}\right) & =-3.3 \times 10^{-4},
\end{aligned}
$$

which are compatible with the most recent experimental data [22, 33]

\section{Factorization and Final state interactions}

The naive factorization has been shown that is unable to reproduce the experimental data on the decays of $D$ and $D_{s}$ mesons into final states with two mesons. Moreover, an important role in this kind of processes is played by the FSI. In the paper [34] the FSI in the decay of $D$ mesons into two pseudoscalar mesons have been studied by assuming, as in [20], that FSI effects are dominated by resonances close to the mass of $D$ mesons. Resonances with isospin 0,1 and $1 / 2$ near the $D$ mass exist and them may contribute to rescatterings among different channels in these respective isospin states and enhance/suppress some of the decay rates. A modified factorization approximation is assumed which is similar to the QCD-factorization in two body decays of $B$ meson [4]. In this case the non factorizable terms are parameterized in the Wilson coefficient and are considered free parameters and fixed by a fit to experimental branching ratios. In [34] the Wexchange and $\mathrm{W}$-annihilation contributions are taken into account and their strengths are obtained from phenomenological fits to the data. The agreement with the available experimental data is quite good (cfr Tables IV, V and VI in ref [34]) but the data on the branching ratios cannot constraint 
the penguin operators contributions and so it is not possible to give predictions on the direct $\mathrm{CP}$ asymmetries in these channels.

However, it is interesting to test the validity of this approach by considering a more general treatment based on $S U(3)_{F}$. In [35, 36, 37], by taking into account high-order perturbation expansion in $S U(3)_{F}$ breaking has been shown by Gronau that some relations between amplitudes receive contributions which are suppressed by powers of $\mathrm{U}$-spin and Isospin breaking parameters. In particular, a class of $D^{0}$ decays related by U-spin symmetry has been identified and relations between their amplitudes are constructed in such a way symmetry breaking terms modify them at fourth order in U-spin and at first order in isospin breaking and second order in U-spin breaking. In fact, if we consider the ratios

$$
\begin{aligned}
R_{1} & \equiv \frac{\left|A\left(D^{0} \rightarrow K^{+} \pi^{-}\right)\right|}{\left|A\left(D^{0} \rightarrow \pi^{+} K^{-}\right)\right| \tan ^{2} \theta_{C}}, \\
R_{2} & \equiv \frac{\left|A\left(D^{0} \rightarrow K^{+} K^{-}\right)\right|}{\left|A\left(D^{0} \rightarrow \pi^{+} \pi^{-}\right)\right|}, \\
R_{3} & \equiv \frac{\left|A\left(D^{0} \rightarrow K^{+} K^{-}\right)\right|+\left|A\left(D^{0} \rightarrow \pi^{+} \pi^{-}\right)\right|}{\left|A\left(D^{0} \rightarrow \pi^{+} K^{-}\right)\right| \tan \theta_{C}+\left|A\left(D^{0} \rightarrow K^{+} \pi^{-}\right)\right| \tan ^{-1} \theta_{C}}, \\
R_{4} & \equiv \sqrt{\frac{\left|A\left(D^{0} \rightarrow K^{+} K^{-}\right)\right|\left|A\left(D^{0} \rightarrow \pi^{+} \pi^{-}\right)\right|}{\left|A\left(D^{0} \rightarrow \pi^{+} K^{-}\right)\right|\left|A\left(D^{0} \rightarrow K^{+} \pi^{-}\right)\right|}},
\end{aligned}
$$

it is possible to show that the $R_{i}$ are not mutually independent and that they obey the following identity

$$
R_{4}=R_{3} \sqrt{\frac{1-\left[\left(R_{2}-1\right) /\left(R_{2}+1\right)\right]^{2}}{1-\left[\left(R_{1}-1\right) /\left(R_{1}+1\right)\right]^{2}}} .
$$

In the limit of $S U(3)_{F} R_{i}=1$ and $\Delta R$ defined by

$$
\Delta R \equiv R_{3}-R_{4}+\frac{1}{8}\left[\left(\sqrt{2 R_{1}-1}-1\right)^{2}-\left(\sqrt{2 R_{2}-1}-1\right)^{2}\right]=\mathscr{O}\left(\varepsilon_{1}^{4}, \varepsilon_{2}^{4}\right)+\mathscr{O}\left(\delta_{1} \varepsilon_{1}^{2}, \delta_{2} \varepsilon_{2}^{2}\right)
$$

is different from zero by terms of the order $\mathscr{O}\left(\varepsilon_{1}^{4}, \varepsilon_{2}^{4}\right)$ and $\mathscr{O}\left(\delta_{1} \varepsilon_{1}^{2}, \delta_{2} \varepsilon_{2}^{2}\right)$, where $\varepsilon_{i}$ and $\delta_{i}$ are U-spin and Isospin breaking terms, respectively. In the case of the results obtained in [34] they have

$$
\Delta R=-0.000013 \pm 0.006 \text {. }
$$

\section{FSI phases as main sources of $S U(3)_{F}$ breaking}

In ref $[20,38]$ large phase differences are obtained by fitting experimental data in the factorization improved approximation for the D meson decays into PS, PP and PV final state. In this approach the large $S U(3)_{F}$ violation are essentially consequences of the large phases. Following the same line of thinking, in [39] we have studied the SCS decays of $D$ mesons into a pair of pseudoscalar mesons by assuming an exact $S U(3)_{F}$ symmetry for the decay amplitudes. The effective hamiltonian for $|\Delta C|=1$ and $\Delta S=0$ transforms as a triplet and so is responsible of the transition of the $D^{0}$ (which is an U-spin singlet) into two different U-spin triplets. The first one is part of the octet representation of $S U(3)_{F}$ the second is in the 27 representation. Thus there are two free 
parameters to be fixed. Concerning the phases, they are related with the Isospin of the final state, and so we have $\delta_{1}$ which is related to the state with $I=1$ in the octet, and $\delta_{0}, \delta_{0}^{\prime}$ and $\phi$ which are related to the $I=0$ in the octet and in the singlet representations; $\phi$ represents the mixing angle. In conclusion, in this approach there are six different free parameters to describe the $D^{0}$ SCS decays and we have shown that a good fit to the data can be obtained [39].

In ref. [40] we are extending the previous approach by considering also the $D^{+}$and $D_{s}$ SCS modes and all the Cabibbo Favoured (CF) and the Double Cabibbo Suppressed (DCS) decay modes. The $S U(3)_{F}$ violation in the CF and DCS decay modes of $D^{0}$ cannot be ascribed only to the strong phase which, in analogy to the previous ones, we called $\delta_{1 / 2}$. Thus, we have introduced a free parameter, $K$, taking into account, in the amplitudes, the non-conservation of the strangeness violating $S U(3)_{F}$ currents. Moreover, the $S U(3)_{F}$ symmetry require the introduction of two new parameters. At the end this approach contains eleven free parameters to describe D meson decays into two pseudoscalar mesons without the $\eta$ and $\eta^{\prime}$ in the final state. Preliminary result of the fit to the experimental branching ratios can be found in the Table 1.

\begin{tabular}{|l|c|l|}
\hline Channel & Fit $\left(\times 10^{-3}\right)$ & Exp. $\left(\times 10^{-3}\right)$ \\
\hline $\mathbf{C F}$ & & \\
\hline $\operatorname{BR}\left(D^{+} \rightarrow \pi^{+} K_{S}\right)$ & $15.72 \pm 0.41$ & $15.3 \pm 0.6$ \\
$\operatorname{BR}\left(D^{+} \rightarrow \pi^{+} K_{L}\right)$ & $14.34 \pm 0.37$ & $14.6 \pm 0.5$ \\
$\operatorname{BR}\left(D^{0} \rightarrow \pi^{+} K^{-}\right)$ & $39.31 \pm 0.40$ & $39.3 \pm 0.4$ \\
$\operatorname{BR}\left(D^{0} \rightarrow \pi^{0} K_{S}\right)$ & $11.9 \pm 0.33$ & $12.0 \pm 0.4$ \\
$\operatorname{BR}\left(D^{0} \rightarrow \pi^{0} K_{L}\right)$ & $9.39 \pm 0.27$ & $10.0 \pm 0.7$ \\
$\operatorname{BR}\left(D_{s}^{+} \rightarrow K^{+} K_{S}\right)$ & $15.0 \pm 0.5$ & $15.0 \pm 0.5$ \\
\hline $\operatorname{SCS}$ & & \\
\hline $\operatorname{BR}\left(D^{0} \rightarrow \pi^{+} \pi^{-}\right)$ & $1.42 \pm 0.03$ & $1.421 \pm 0.025$ \\
$\operatorname{BR}\left(D_{0}^{+} \rightarrow \pi^{0} \pi^{0}\right)$ & $0.83 \pm 0.04$ & $0.826 \pm 0.035$ \\
$\operatorname{BR}\left(D^{+} \rightarrow \pi^{+} \pi^{0}\right)$ & $1.22 \pm 0.06$ & $1.24 \pm 0.06$ \\
$\operatorname{BR}\left(D^{0} \rightarrow K^{+} K^{-}\right)$ & $4.02 \pm 0.06$ & $4.01 \pm 0.07$ \\
$\operatorname{BR}\left(D^{0} \rightarrow K_{S} K_{S}\right)$ & $0.17 \pm 0.04$ & $0.18 \pm 0.04$ \\
$\operatorname{BR}\left(D^{+} \rightarrow K^{+} K_{S}\right)$ & $2.89 \pm 0.12$ & $2.95 \pm 0.15$ \\
$\operatorname{BR}\left(D_{s}^{+} \rightarrow \pi^{0} K^{+}\right)$ & $1.03 \pm 0.04$ & $0.63 \pm 0.21$ \\
$\operatorname{BR}\left(D_{s}^{+} \rightarrow \pi^{+} K_{S}\right)$ & $1.24 \pm 0.06$ & $1.22 \pm 0.06$ \\
\hline $\operatorname{DCS}$ & & \\
\hline $\operatorname{BR}\left(D^{+} \rightarrow \pi^{0} K^{+}\right)$ & $0.155 \pm 0.005$ & $0.189 \pm 0.025$ \\
$\operatorname{BR}\left(D^{0} \rightarrow \pi^{-} K^{+}\right)$ & $0.140 \pm 0.003$ & $0.1399 \pm 0.0027$ \\
\hline
\end{tabular}

Table 1: In the second column the branching ratios used in the fit, in the last column the predictions for the values obtained for the fitted values of the parameters.

The test suggested by Gronau [37] gives, for this model, $\Delta R=0.0030 \pm 0.0004$ (cfr. eq. (5.3)).

The $\mathrm{CP}$ violating part of the amplitudes, in this approach, depend essentially on two unknown parameters that cannot be determined by the branching ratios. However, they can be extracted from the experimental data on $\Delta a_{\mathrm{CP}}$ and so predictions of $\mathrm{CP}$ asymmetries for final state with kaons and 
pions can be done [40].

7. $a_{\mathrm{CP}}\left(D^{0} \rightarrow K_{S} K_{S}\right)$

The decay mode $D^{0} \rightarrow K^{0} \bar{K}^{0}$ is interesting because, as was pointed out many years ago in [20], the tree level amplitude $A\left(D^{0} \rightarrow K^{0} \bar{K}^{0}\right)$ vanish in the limit of $S U(3)_{F}$ but this is not true if we look at the penguin contributions. The fact that the ratio of the penguin to the tree amplitude could be large implies that the CP asymmetry (cfr eq. (1.2)) may be enhanced at observable level [41]. The penguin annihilation term and the exchange contribution (relevant for the calculation) are estimated by a perturbative approach and by using the results of a fit to all $\mathrm{D}$ decays in two pseudoscalar mesons, respectively. They give [41]

$$
\left|a_{\mathrm{CP}}\left(D^{0} \rightarrow K_{S} K_{S}\right)\right| \leqslant 1.1 \% \quad(95 \% \text { C.L. }) .
$$

In a previous analysis [42] the upper bound

$$
\left|a_{\mathrm{CP}}\left(D^{0} \rightarrow K_{S} K_{S}\right)\right| \lesssim \frac{2\left|V_{c b} V_{u b}\right|}{\varepsilon\left|V_{c s} V_{u s}\right|} \sim 0.6 \%,
$$

is obtained which is in fairly good agreement with eq. (7.1). Here, $\varepsilon$ is a measure of the $S U(3)_{F}$ breaking. Finally, by assuming similar magnitude for two different matrix elements in [23] the relation

$$
\left|a_{\mathrm{CP}}\left(D^{0} \rightarrow K_{S} K_{S}\right)\right| \lesssim \frac{3}{2} \Delta a_{\mathrm{CP}}
$$

has been given. However, this prediction cannot be considered reliable because, as stressed in [41], $a_{\mathrm{CP}}\left(D^{0} \rightarrow K_{S} K_{S}\right)$ and $\Delta a_{\mathrm{CP}}$ involve different topological amplitudes.

From the experimental point of view, we have the following measurements

$$
\begin{array}{ll}
a_{\mathrm{CP}}\left(D^{0} \rightarrow K_{S} K_{S}\right)=(-23 \pm 19) \%, & \operatorname{CLEO}(2001)[43] \\
a_{\mathrm{CP}}\left(D^{0} \rightarrow K_{S} K_{S}\right)=(-2.9 \pm 5.2 \pm 2.2) \%, & \operatorname{LHCb}(2015)[44] \\
a_{\mathrm{CP}}\left(D^{0} \rightarrow K_{S} K_{S}\right)=(-0.2 \pm 1.53 \pm 0.17) \% & \text { Belle }(2016)[45]
\end{array}
$$

and so the asymmetry is compatible with zero.

\section{Summary}

We have reviewed approaches to study the nonleptonic decays of $D$ and $D_{s}$ mesons into two pseudoscalar mesons. We have seen that the use of the $S U(3)_{F}$ symmetry alone, even if it allows a consistent description of the branching ratios when symmetry breaking is considered, cannot give predictions on the $\mathrm{CP}$ asymmetries, due to the large number of RME. However, physical considerations and/or some other approximations (like, for example, improved factorization) can, together with a model to take into account FSI, give not only a good description of the measured branching fractions but also reliable predictions on $\mathrm{CP}$ asymmetries.

Acknowledgments: It is a pleasure to thank F. Buccella, E. Franco, M. Lusignoli, A. Paul, A. Pugliese and L. Silvestrini for collaboration and discussions. Many thanks also to A. Carbone for discussions. 


\section{References}

[1] A. Lenz, arXiv:1610.07943 [hep-ph].

[2] Ayan Paul, these proceedings; talk at CHARM 2016.

[3] B. Loiseau, arXiv:1611.05286 [hep-ph].

[4] M. Beneke, G. Buchalla, M. Neubert and C. T. Sachrajda, Phys. Rev. Lett. 83 (1999) 1914 [hep-ph/9905312].

[5] Guido Martinelli, these proceedings; talk at CHARM 2016.

[6] Marco Ciuchini, these proceedings; talk at CHARM 2016.

[7] G. Altarelli, N. Cabibbo and L. Maiani, Nucl. Phys. B 88 (1975) 285.

[8] R. L. Kingsley, S. B. Treiman, F. Wilczek and A. Zee, Phys. Rev. D 11 (1975) 1919.

[9] R. L. Kingsley, S. B. Treiman, F. A. Wilczek and A. Zee, Phys. Rev. D 12 (1975) 106.

[10] C. Quigg, Z. Phys. C 4 (1980) 55.

[11] T. Aaltonen et al. [CDF Collaboration], Phys. Rev. Lett. 109 (2012) 111801 [arXiv:1207.2158 [hep-ex]].

[12] R. Aaij et al. [LHCb Collaboration], Phys. Rev. Lett. 108 (2012) 111602 [arXiv:1112.0938 [hep-ex]].

[13] B. R. Ko [Belle Collaboration], PoS ICHEP 2012 (2013) 353 [arXiv:1212.1975 [hep-ex]].

[14] B. Aubert et al. [BaBar Collaboration], Phys. Rev. Lett. 100 (2008) 061803 [arXiv:0709.2715 [hep-ex]].

[15] R. Aaij et al. [LHCb Collaboration], Phys. Lett. B 723 (2013) 33 [arXiv:1303.2614 [hep-ex]].

[16] R. Aaij et al. [LHCb Collaboration], JHEP 1407 (2014) 041 [arXiv:1405.2797 [hep-ex]].

[17] R. Aaij et al. [LHCb Collaboration], JHEP 1504 (2015) 043 [arXiv:1501.06777 [hep-ex]].

[18] Y. Grossman, A. L. Kagan and Y. Nir, Phys. Rev. D 75 (2007) 036008 [hep-ph/0609178]; G. Isidori, J. F. Kamenik, Z. Ligeti and G. Perez, Phys. Lett. B 711 (2012) 46 [arXiv:1111.4987 [hep-ph]];

K. Wang and G. Zhu, Phys. Lett. B 709 (2012) 362 [arXiv:1111.5196 [hep-ph]];

G. Hiller, Y. Hochberg and Y. Nir, Phys. Rev. D 85 (2012) 116008 [arXiv:1204.1046 [hep-ph]];

G. F. Giudice, G. Isidori and P. Paradisi, JHEP 1204 (2012) 060 [arXiv:1201.6204 [hep-ph]];

W. Altmannshofer, R. Primulando, C. T. Yu and F. Yu, JHEP 1204 (2012) 049 [arXiv:1202.2866 [hep-ph]];

C. H. Chen, C. Q. Geng and W. Wang, Phys. Rev. D 85 (2012) 077702 [arXiv:1202.3300 [hep-ph]];

O. Gedalia, J. F. Kamenik, Z. Ligeti and G. Perez, Phys. Lett. B 714 (2012) 55 [arXiv:1202.5038 [hep-ph]];

T. Mannel and N. Uraltsev, JHEP 1303 (2013) 064 [arXiv:1205.0233 [hep-ph]];

B. Keren-Zur, P. Lodone, M. Nardecchia, D. Pappadopulo, R. Rattazzi and L. Vecchi, Nucl. Phys. B 867 (2013) 394 [arXiv:1205.5803 [hep-ph]];

R. Barbieri, D. Buttazzo, F. Sala and D. M. Straub, JHEP 1210 (2012) 040 [arXiv:1206.1327

[hep-ph]]; 
A. D. Dolgov, S. I. Godunov, A. N. Rozanov and M. I. Vysotsky, JETP Lett. 96 (2012) 290 [arXiv:1206.6652 [hep-ph]];

C. Delaunay, J. F. Kamenik, G. Perez and L. Randall, JHEP 1301 (2013) 027 [arXiv:1207.0474 [hep-ph]].

[19] M. Golden and B. Grinstein, Phys. Lett. B 222 (1989) 501;

D. Pirtskhalava and P. Uttayarat, Phys. Lett. B 712 (2012) 81 [arXiv:1112.5451 [hep-ph]];

B. Bhattacharya, M. Gronau and J. L. Rosner, Phys. Rev. D 85 (2012) 054014 [arXiv:1201.2351 [hep-ph]];

T. Feldmann, S. Nandi and A. Soni, JHEP 1206 (2012) 007 [arXiv:1202.3795 [hep-ph]];

J. Brod, Y. Grossman, A. L. Kagan and J. Zupan, JHEP 1210 (2012) 161 [arXiv:1203.6659 [hep-ph]];

H. -Y. Cheng and C. -W. Chiang, Phys. Rev. D 86 (2012) 014014 [arXiv:1205.0580 [hep-ph]]. See also [21, 23, 39, 42]

[20] F. Buccella, M. Lusignoli, G. Miele, A. Pugliese and P. Santorelli, Phys. Rev. D 51 (1995) 3478 [hep-ph/9411286].

[21] E. Franco, S. Mishima and L. Silvestrini, JHEP 1205 (2012) 140 [arXiv:1203.3131 [hep-ph]].

[22] C. Patrignani et al. [Particle Data Group Collaboration], Chin. Phys. C 40 (2016) no.10, 100001.

[23] G. Hiller, M. Jung and S. Schacht, Phys. Rev. D 87 (2013) no.1, 014024 [arXiv:1211.3734 [hep-ph]].

[24] G. Hiller, M. Jung and S. Schacht, PoS EPS -HEP2013 (2013) 371 [arXiv:1311.3883 [hep-ph]].

[25] L. L. Chau, Phys. Rept. 95 (1983) 1.

[26] L. L. Chau and H. Y. Cheng, Phys. Rev. Lett. 56 (1986) 1655.

[27] L. L. Chau and H. Y. Cheng, Phys. Rev. D 36 (1987) 137.

[28] L. L. Chau and H. Y. Cheng, Phys. Lett. B 280 (1992) 281.

[29] S. Müller, U. Nierste and S. Schacht, Phys. Rev. D 92 (2015) no.1, 014004 [arXiv:1503.06759 [hep-ph]].

[30] S. Müller, U. Nierste and S. Schacht, Phys. Rev. Lett. 115 (2015) no.25, 251802 [arXiv:1506.04121 [hep-ph]].

[31] R. Dhir, C. S. Kim and S. Oh, Int. J. Mod. Phys. A 30 (2015) no.17, 1550104 [arXiv:1504.02556 [hep-ph]].

[32] H. Y. Cheng and C. W. Chiang, Phys. Rev. D 85 (2012) 034036 Erratum: [Phys. Rev. D 85 (2012) 079903] [arXiv:1201.0785 [hep-ph]].

[33] Y. Amhis et al. [Heavy Flavor Averaging Group (HFAG) Collaboration], arXiv:1412.7515 [hep-ex].

[34] A. Biswas, N. Sinha and G. Abbas, Phys. Rev. D 92 (2015) no.1, 014032 [arXiv:1503.08176 [hep-ph]].

[35] M. Gronau, Phys. Lett. B 730 (2014) 221 Addendum: [Phys. Lett. B 735 (2014) 282] [arXiv:1311.1434 [hep-ph]].

[36] M. Gronau, Phys. Rev. D 90 (2014) no.11, 117901 [arXiv:1410.7255 [hep-ph]].

[37] M. Gronau, Phys. Rev. D 91 (2015) no.7, 076007 [arXiv:1501.03272 [hep-ph]].

[38] F. Buccella, M. Lusignoli and A. Pugliese, Phys. Lett. B 379 (1996) 249 [hep-ph/9601343]. 
[39] F. Buccella, M. Lusignoli, A. Pugliese and P. Santorelli, Phys. Rev. D 88 (2013) no.7, 074011 [arXiv:1305.7343 [hep-ph]].

[40] F. Buccella, E. Franco, A. Paul, P. Santorelli and L. Silvestrini, in preparation.

[41] U. Nierste and S. Schacht, Phys. Rev. D 92 (2015) no.5, 054036 [arXiv:1508.00074 [hep-ph]].

[42] J. Brod, A. L. Kagan and J. Zupan, Phys. Rev. D 86 (2012) 014023 [arXiv:1111.5000 [hep-ph]].

[43] G. Bonvicini et al. [CLEO Collaboration], Phys. Rev. D 63 (2001) 071101 [hep-ex/0012054].

[44] R. Aaij et al. [LHCb Collaboration], JHEP 1510 (2015) 055 [arXiv:1508.06087 [hep-ex]].

[45] A. Abdesselam et al., arXiv:1609.06393 [hep-ex]. 